\title{
DEFESA E FRAUDE ALIMENTAR NA INTE- GRIDADE DA CADEIA DE FORNECIMENTO DE ALIMENTOS - BREVE REVISÃO
}

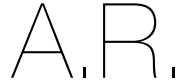
ARTIGO DE REVISÃO

1 Centro de Biotecnologia e Química Fina Laboratório Associado da Escola Superior de Biotecnologia da Universidade Católica Portuguesa,

Rua Diogo Botelho, n. ${ }^{\circ}$ 1327 ,

4169-005 Porto, Portugal

Endereço para correspondência:

Maria Joāo Costa Rua Diogo Botelho, n. ${ }^{\circ} 1327$ 4169-005 Porto, Portugal maria_joao_costa10@hotmail.con

Histórico do artigo:

Recebido a 9 de maio de 2019 Aceite 31

\section{FOOD DEFENSE AND FOOD FRAUD IN FOOD CHAIN INTEGRITY - BRIEF REVIEW}

RESUMO

A Organização Mundial da Saúde identificou a contaminação alimentar intencional como uma das principais ameaças à saúde pública no século XXI, cujos impactos podem assumir proporções consideráveis na saúde dos consumidores, sociedade, economia, política e segurança nacional.

A temática da defesa alimentar é recente em Portugal e a sua implementação têm-se concentrado em empresas detentoras de referenciais suportados pela Global Food Safety Initiative.

Este trabalho tem como objetivo apresentar os conceitos de defesa alimentar, fraude alimentar e proteção alimentar, o seu contributo para a integridade da cadeia de fornecimento de alimentos bem como as principais metodologias reconhecidas para responder à temática.

A defesa alimentar contribui para a mitigação de riscos potenciais em contaminação intencional e fraude alimentar. Existem várias metodologias e ferramentas que auxiliam na implementação de um sistema de defesa alimentar, sendo que a maioria tem por base o sistema HACCP (Hazard Analysis and Critical Control Points).

A implementação de sistemas de defesa alimentar e de prevenção da fraude alimentar acrescentam robustez e coesão à cadeia de fornecimento de alimentos, sendo, por isso, essencial aumentar a consciencialização dos profissionais e operadores do setor alimentar para o tema.

\section{PALAVRAS-CHAVE}

Ameaça, Defesa alimentar, Fraude alimentar, Proteção alimentar, Vulnerabilidade

ABSTRACT

The World Health Organization has identified intentional food contamination as one of the major threats to public health in the $21^{\text {st }}$ century, which can affect substantially consumer health, society, the economy, politics, and national security.

Food defense is recent in Portugal and its implementation has focused on companies holding references supported by the Global Food Safety Initiative.

This paper aims to present the concepts of food defense, food fraud, and food protection, their contribution to the integrity of the food supply chain as well as the main recognized methodologies to respond to the subject.

Food defense contributes to mitigating risks applicable to intentional contamination and food fraud. Some several methodologies and tools assist in the implementation of a food defense system, most of which are based on the HACCP system (Hazard Analysis and Critical Control Points).

The implementation of food defense and food fraud prevention systems adds robustness and cohesion to the food supply chain; therefore, it is essential to raise the awareness of food professionals and food operators.

KEYWORDS

Threat, Food defense, Food fraud, Food integrity, Vulnerability

\section{INTRODUÇÃO}

Nos dias de hoje, o terrorismo no mundo ocidental é uma preocupação constante, tendo mesmo a Organização Mundial da Saúde (OMS) identificado a contaminação alimentar intencional como uma das principais ameaças à saúde pública do século XXI (1).

A cadeia de fornecimento de alimentos surge como um alvo fácil para um ataque e apresenta baixa segurança

(2). A contaminação intencional de alimentos pode ter consequências significativas para a sociedade e saúde pública como doença, morte e o medo generalizado na população. A nível económico, os impactos podem ser também bastantes devastadores, com a interrupção da cadeia de abastecimento ou quebras na exportação, aliados à perda de confiança do público na segurança dos alimentos e na eficácia dos governos (3).

As entidades governamentais e organizações internacionais, preocupam-se cada vez mais com a contaminação 
intencional e criminosa da cadeia de fornecimento de alimentos e com as suas consequências (4).

A primeira medida legislativa teve origem nos Estados Unidos da América em 2002 com o "Public Health Security and Bioterrorism Preparedness and Response Act (Bioterrorism Act 2002)". A evolução da temática neste país tem sido largamente intensificada. Em 2016, $85 \%$ dos estabelecimentos industriais americanos ostentavam planos funcionais de defesa alimentar (5).

No lado Asiático, a aprovação voluntária de um conjunto de "Princípios Defesa Alimentar" por todos os 21 membros da Asia-Pacific Economic Cooperation (APEC) em 2007, veio também reforçar a consciencialização sobre o tema (4).

Na Europa, os primeiros desenvolvimentos surgiram no Reino Unido, refletidos no documento Publically Available Specification (PAS) 96, elaborado pela British Standards Institution (BSI), cuja primeira versão data de 2008 (6). Em oposição aos Estados Unidos, em muitos países da União Europeia, a defesa alimentar é ainda um conceito bastante novo (7).

Ao nível das organizações, as empresas do tecido agroalimentar corroboram também com esta inquietação e têm vindo a integrar nos seus Sistemas de Gestão de Segurança Alimentar, medidas de defesa alimentar. Por um lado, esta implementação surge como resposta aos requisitos de defesa alimentar presentes nos referenciais reconhecidos pela Global Food Safety Initiative (GFSI) como é o caso do International Featured Standards (IFS), British Retail Consortium (BRC) e Food Safety System Certification (FSSC) 22000 e, nesta ótica, as empresas certificadas por um ou mais destes referenciais, concedem o devido cumprimento aos seus requisitos. Por outro lado, outras organizações e serviços de alimentação, não detentoras de referenciais apoiados pela GFSI, optam igualmente por implementar um sistema de defesa alimentar como resposta ao contexto atual, por forma a demonstrar que também tomam os cuidados necessários na proteção dos seus produtos e instalações, possibilitando também satisfazer as exigências dos seus clientes e parceiros comerciais (6). Na União Europeia, os requisitos legislativos concentram-se principalmente na segurança alimentar. Não há regulamentação que implique a implementação de um sistema de defesa alimentar, sendo que, as empresas e serviços de alimentação que o fazem, implementam-no a título voluntário.

Já no que respeita à fraude alimentar esta encontra-se referenciada na legislação europeia e nacional no que respeita às práticas fraudulentas e enganosas que podem ter um impacto direto ou indireto na segurança dos géneros alimentícios $(8,9)$.

Todas as sociedades são fundamentalmente dependentes do fornecimento de alimentos, portanto, a sua interrupção pode constituir um objetivo óbvio para o terrorismo (10). Esta nova realidade, fomentou o desenvolvimento da defesa alimentar como uma área autónoma de estudo de um nova disciplina: a proteção alimentar (11).

\section{Proteção Alimentar, Defesa Alimentar e Fraude Alimentar}

O conceito de proteção alimentar (food protection) inclui a combinação de qualidade alimentar (food quality), segurança dos alimentos (food safety), fraude alimentar (food fraud) e defesa alimentar (food defense) (11). A falta de qualidade alimentar é uma ameaça económica, que não é intencional. A fraude alimentar tem motivação económica e intencional, mas não se destina a ameaçar a saúde pública (11). Já um incidente de segurança alimentar é um ato não intencional com dano involuntário, enquanto que um ataque que afeta a defesa alimentar é um ato intencional com dano intencional. Várias entidades têm emitidos as suas definições de defesa alimentar. A British Standards Institution (BSI) define defesa alimentar como "procedimentos adotados para garantir a segurança de alimentos, bebidas e as suas cadeias de abastecimento de ataques maliciosos e ideologicamente motivados que levam à contaminação ou à interrupção do fornecimento" (6). O conceito de defesa alimentar engloba ainda o bioterrorismo e antiterrorismo (12). Importa referir que, por detrás de um ato de contaminação intencional está sempre pelo menos um indivíduo, ou seja, qualquer que seja o ataque, forma e/ou consequência, ocorre porque houve intervenção humana intencional.

Já a fraude alimentar, incluindo a subcategoria, adulteração economicamente motivada, é um risco alimentar que ganha cada vez mais destaque (11). A Comissão Europeia sentiu a necessidade de criar recentemente o Centro de Conhecimento sobre Fraude Alimentar e Qualidade dos Alimentos, que proporcionará aos responsáveis políticos da União Europeia assim como às autoridades nacionais, acesso e conhecimento científico atualizado sobre questões de fraude e qualidade alimentares (13).

Fraude alimentar é "um termo coletivo usado para englobar a substituição deliberada e intencional, a adição, adulteração ou falsificação de alimentos, ingredientes alimentícios ou embalagens de alimentos; ou declarações falsas ou enganosas feitas sobre um produto, para ganhos económicos" (11).

A adulteração economicamente motivada terá, por definição, uma motivação económica, mas os riscos para a saúde pública são muitas vezes maiores do que as ameaças tradicionais de segurança alimentar porque os contaminantes usados não são os convencionais (11). De acordo com a Food Standards Agency (FSA) e em particular The National Food Crime Unit, a fraude alimentar torna-se crime alimentar sempre que a escala e o impacto potencial da atividade são tidos como sérios, cuja atividade criminosa tem um alcance regional, nacional ou internacional, um risco significativo para a segurança pública, ou perda financeira substancial para consumidores ou empresas (14). A extensão total e o impacto da criminalidade alimentar podem não ser imediatamente percebidos (14). As áreas de criminologia e ciências comportamentais proporcionam uma visão mais abrangente da motivação e causalidade do crime alimentar (15).

Fraude alimentar geralmente é mais fácil para alimentos líquidos do que para sólidos, assim como para alimentos com vários ingredientes comparativamente aos mais simples, de ingrediente único (16). Cadeias de fornecimento mais longas resultam também em maior risco de fraude alimentar (16).

De acordo com informação da Autoridade de Segurança Alimentar e Económica (ASAE), no top 10 dos produtos fraudulentos, no período de 2015-2016, encontra-se o azeite devido principalmente à mistura de óleos, seguido dos produtos pré-cozinhados à base de carne ou peixe nos quais houve substituição da espécie, margarinas, pelo uso de gorduras diferentes das especificadas, hortofrutícolas frescos devido à alteração da origem, queijos e vinhos devido à adulteração e contrafação, pescado e bacalhau devido à substituição da espécie e ainda os suplementos alimentares pelo substituição/adulteração de ingredientes (17).

Apesar da defesa alimentar ter como foco, proteger os alimentos de contaminações destinadas a provocar danos na saúde publica, danos económicos, medo e terror e a fraude alimentar ter como motivação o ganho económico, os temas fundem-se parcialmente quando é para desenvolver um sistema de prevenção das contaminações intencionais (18).

\section{Os Alimentos como Veículo de Ato Terrorista}

Ao longo da história, foram relatados vários casos em que o abastecimento de alimentos foi sabotado deliberadamente, durante 
campanhas militares e, mais recentemente, para aterrorizar ou de outra forma, intimidar as populações civis (19).

A contaminação deliberada de alimentos pode ocorrer em qualquer ponto vulnerável ao longo da cadeia de fornecimento de alimentos, do prado ao prato, dependendo do alimento e do agente(19). De acordo com a Food and Drug Administration (FDA), os três componentes de uma operação de ataque a sistemas de alimentação e água são (20): 1. Agressores: criminosos, concorrentes, terroristas, ativistas e colaboradores desonestos ou descontentes;

2. Táticas usadas pelos agressores: as táticas podem incluir os ataques externos, entrada forçada, uso de credenciais falsas, uso de alguém com acesso legítimo, entre outros;

3. Agentes usados por um agressor: agentes biológicos (bactérias, toxinas, vírus, parasitas, etc.) sob a forma de líquidos, aerossóis ou sólidos; agentes químicos sob a forma de gotículas, líquidos, aerossóis ou sólidos que podem incluir agentes clássicos de guerra química ou produtos químicos industriais tóxicos (pesticidas, rodenticidas e metais pesados); agentes radiológicos, elementos radioativos em forma líquida ou sólida; agentes físicos, de que são exemplo, fragmentos de osso, fragmentos de vidro e limalhas de metal.

O relatório técnico da Nanyang Technological University narra os incidentes de contaminação maliciosa, "do prado ao prato", ocorridos entre 1950 e 2008. Este relatório incidiu sobre mais de 450 incidentes e revelou que cerca de $98 \%$ dos incidentes ocorreram a jusante na cadeia de fornecimento, ou seja, nas lojas de retalho, em casa e no local de trabalho, porém, os casos que tiveram lugar nos serviços de alimentação resultaram num maior impacto na saúde pública (4). Este relatório evidenciou também que os agentes mais utilizados foram produtos químicos domésticos, agrícolas ou industriais comumente disponíveis, e com os quais os perpetradores frequentemente trabalhavam, dispondo assim de conhecimento e acesso aos mesmos. Já no que respeita à água, o total de sete casos encontrados, sugere que é muito difícil contaminar intencionalmente o abastecimento de água em grande escala e com grande impacto na saúde pública (4).

\section{Sistemas de Gestão da Segurança Alimentar- Metodologias}

O desenvolvimento de um modelo de avaliação de risco para um ataque terrorista à cadeia de fornecimento de alimentos deve considerar três aspetos: a capacidade do terrorista para conduzir o ataque, a intenção do terrorista para executar o ataque e a oportunidade para conduzir o ataque. Estes fatores criam a ameaça. O risco é a ameaça associada à vulnerabilidade. A vulnerabilidade é a acessibilidade ao alimento ou cadeia alvo ao ataque (10).

A capacidade de quantificar a probabilidade de uma ameaça ou vulnerabilidade numa determinada situação é de muitas maneiras influenciada pelo grau de adoção de contramedidas e sua eficácia (15). De acordo com o United States Department of Agriculture (USDA), os elementos para um plano de defesa alimentar funcional passam por desenvolver, escrever, implementar, testar, avaliar, e manter um plano de defesa alimentar (21). Para suprimir todas estas etapas, à semelhança do que acontece num sistema HACCP, é crucial a existência de uma equipa de trabalho - equipa de defesa alimentar - que coordene todo este desenvolvimento, implementação e manutenção. Tendo em conta que os agentes, veículos, acessos, motivações e implicações para um ataque podem ser tão diversos e disseminados por várias áreas de conhecimento, uma equipa multidisciplinar constitui a opção de referência.

Várias metodologias têm vindo a ser propostas para avaliação de risco e vulnerabilidade na defesa alimentar e na fraude alimentar. Operacional Risk Management (ORM), Hazard Analysis and Risk
Based Preventive Controls (HARPC), Hazard Analysis Critical Control and Defense Point (HACCDP), Vulnerability Assesssment and Critical Control Points (VACCP), CARVER+Shock, Threat Assessment Critical Control Points (TACCP) são exemplos de metodologias adaptadas ou criadas de raiz para responder à prevenção da contaminação e da adulteração intencional $(18,20,22-24)$. Cada organização deve adotar a metodologia que considera mais adequada à sua realidade, área de atividade, dimensão e características.

Na perspetiva da GFSI, a Gestão do Sistema de Segurança Alimentar engloba o HACCP (risco / segurança alimentar), o TACCP (ameaça / defesa alimentar) e o VACCP (vulnerabilidade / fraude alimentar). Para a GFSI, estes são definidos como três pilares separados que necessitam de ser abordados individualmente (Figura 1) (25).

\section{Figura 1}

Sistema de Gestão da Segurança Alimentar da GFSI (adaptado de Spink, 2014 (25))

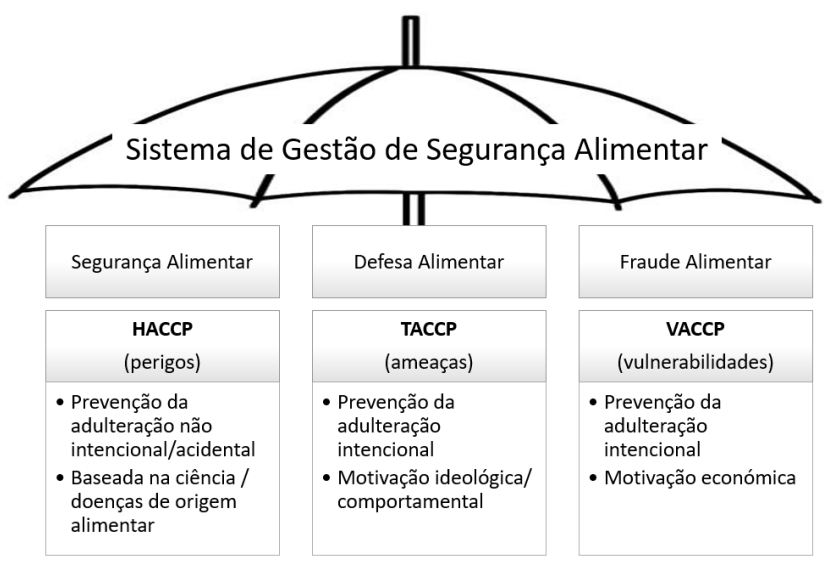

HACCP: Hazard Analysis and Critical Control Points TACCP:Threat Assessment Critical Control Points VACCP: Vulnerability Assessment and Critical Control Point System

\section{Metodologia "Vulnerability Assessment and Critical Control Point System" - VACCP}

VACCP tem como propósito avaliar o quão suscetível e/ou exposta a organização está à fraude alimentar. O objetivo é a prevenção da adulteração intencional economicamente motivada (15). O Conselho da GFSI decidiu seguir as recomendações do painel de reflexão sobre fraude alimentar - "Food Fraud Think Tank"- e propõe, no documento "GFSI Position On Mitigating The Public Health Risk Of Food Fraud" incorporar duas etapas de mitigação fraude alimentar na forma de dois elementos-chave (26):

1. Avaliação de vulnerabilidade à fraude alimentar - colher informações nos vários pontos ao longo da cadeia de fornecimento de alimentos, incluindo matérias-primas, ingredientes, produtos, embalagens, e avaliar as mesmas, de modo a identificar e priorizar as vulnerabilidades significativas;

2. Ter um plano de controlo - implementar medidas de controlo adequadas para reduzir os riscos dessas vulnerabilidades, medidas que podem incluir uma estratégia de monitorização, testes, verificação de origem, especificações, auditorias de fornecedores ou tecnologias anti contrafação. Um plano de controlo claramente documentado descreve quando, onde e como mitigar atividades fraudulentas. Food Fraud Vulnerability Assessment Tool do SSAFE, parceiro da GFSI é uma das ferramentas de apoio criadas para auxiliar a avaliação de vulnerabilidades (27). 
Metodologia "Threat Assessment Critical Control Points" - TACCP A metodologia TACCP descrita na "PAS 96: Guide to protecting and defending food and drink from deliberate attack" tem como objetivo garantir a autenticidade dos alimentos, pela minimização da oportunidade de um ataque bem como a atenuação das consequências de um ataque já ocorrido (6).

Podendo ser adotada por empresas da área alimentar de todas as dimensões e pontos da cadeia de fornecimento de alimentos, a TACCP apresenta-se como uma metodologia de gestão de risco, que está alinhada com o HACCP, mas apresenta um foco diferente. Esse foco são as pessoas (6).

TACCP define-se como a "Gestão sistemática do risco através da avaliação de ameaças, identificação de vulnerabilidades e implementação de controlos em produtos e materiais, compras, processos, infraestruturas, redes de distribuição e sistemas de negócio por uma equipa experiente e confiável com autoridade para implementar mudanças nos procedimentos" (6). Na implementação de um sistema TACCP, o desafio proposto à equipa TACCP é o de pensar como um atacante e deste modo conseguir antecipar as suas motivações e habilidades (6).

Devem ser percorridas 15 etapas, conforme descritas na Figura 2. No que respeita à avaliação de ameaças, a equipa TACCP deve considerar fornecedores sob stresse financeiro, funcionários alienados e ex-funcionários, concorrentes comerciais, organizações media, organizações terroristas, criminosos e grupos de pressão locais. A adulteração economicamente motivada, a contaminação maliciosa, a extrusão, espionagem, contrafação e crime cibernético são tipos de ameaças que as organizações podem enfrentar. A organização, processo e produto podem ser alvo de um ataque por parte de pessoa singular ou grupos de pessoas e como tal, as suas avaliações devem ser efetuadas separadamente (6).

No que respeita à avaliação de vulnerabilidades e etapas-chave, estas variam de organização para organização de acordo com o negócio e o contexto, porém há dois aspetos que devem ser destacados: a adulteração economicamente motivada e a contaminação maliciosa.
O controlo de acessos, as barreiras físicas, sistemas de deteção de sabotagem e garantias de segurança com o pessoal são controlos críticos que assumem a merecida importância (6).

\section{ANÁLISE CRÍTICA}

A consciência da defesa alimentar não é a mesma em todas as partes do mundo. No entanto, o mundo é uma aldeia global com alimentos e bebidas no mercado global e, nesta ótica, o risco de propagação de alimentos contaminados e adulterados é elevado (7). Esta consciência de ameaça assim como os casos mediáticos de fraude alimentar fomentam a necessidade de aumento de segurança nas cadeias de fornecimento de alimentos (28). Enquanto nos Estados Unidos, a implementação de medidas relativas à defesa alimentar evoluiu consideravelmente, na Europa apenas recentemente a temática tem vindo a avançar.

Várias entidades governativas e/ou institucionais emitiram as suas próprias definições sobre defesa alimentar, fraude alimentar, qualidade alimentar e bioterrorismo assim como conceitos associados como risco, vulnerabilidade e ameaça. Todas as pluralidades de definições geram confusão e interpretações diversas na abordagem ao desenvolvimento e implementação de sistemas de defesa alimentar. Alguns estudos recentes, têm apresentado o entendimento geral das terminologias e da variedade de definições atualmente em uso, de modo a proporcionar orientação para a clarificação e harmonização de conceitos $(29,30)$. Um dos passos futuros poderá passar por um diálogo internacional das diversas entidades no sentido do alinhamento dos termos de uso e suas definições (30).

No que concerne às metodologias VACCP e TACCP apoiadas pela GFSI, verifica-se que derivam do sistema HACCP, o que traz vantagens por serem já do conhecimento dos operadores alimentares e pela facilidade de integração com as práticas existentes para proteger a segurança dos alimentos. Por outro lado, nestas metodologias é essencial compreender o atacante, no que respeita às suas motivações, capacidade e oportunidade, o que poderá não ser tão familiar para os operadores alimentares. As organizações tendem a focar-se em

\section{Figura 2}

Etapas para desenvolvimento e implementação do TACCP (adaptado de BSI- PAS 96:2017(6))
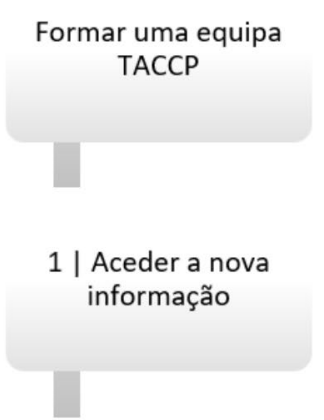

2| Identificar e avaliar ameaças para a organização

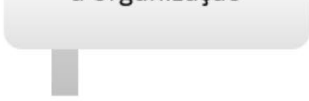

3| Identificar e avaliar as ameaças para a operação

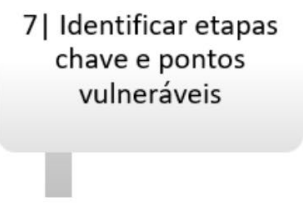

\section{6| Desenhar}

fluxograma da cadeia de fornecimento do produto

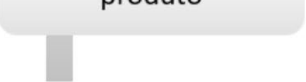

5| Identificar e avaliar ameaças para o produto

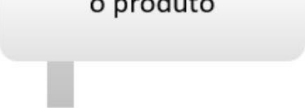

4| Decidir produto sistema
8| Considerar o impacto das ameaças identificadas

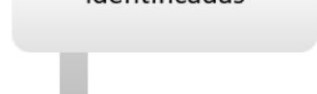

9| identificar os pontos de fornecimento mais críticos

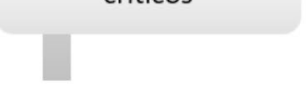

10| Determinar se os procedimentos de controlo detetam a ameaça

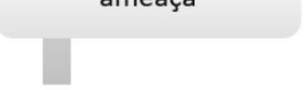

11| Probabilidade vs Impacto - Prioridades
15 | Horizontal Scan e riscos emergentes

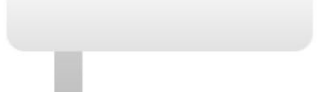

14| Rever e auditar o plano

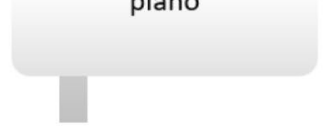

13| Decidir e implementar controlos

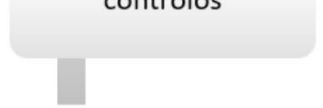

12| Identificar quem pode conduzir a ameaça 
elementos externos à organização e podem não considerar que o atacante seja alguém próximo e/ou da própria organização $(6,15)$. Por conseguinte, estes potenciais agressores não são tidos em conta na avaliação de ameaças e vulnerabilidades da organização, afetando a identificação de prioridades e por fim a eficácia dos sistemas VACCP e TACCP.

Qualquer que seja a metodologia adotada para implementação de um sistema de defesa alimentar, de forma isolada ou combinada, nenhuma consegue garantir que a organização não será alvo de um ataque deliberado malicioso. A existência de um sistema de rastreabilidade, conforme requisito legal, possibilita rastrear e recuperar os produtos afetados (8). No entanto, as organizações devem também prever as suas formas de atuação, no caso de ataque ocorrer ou ter já ocorrido. É mais fácil e económico a prevenção de um incidente, do que que lidar com as consequências de uma contaminação ou adulteração em alarga escala (31). A defesa alimentar e fraude alimentar incrementam integridade na cadeia de fornecimento de alimentos.

\section{CONCLUSÕES}

Com cadeias de fornecimento de alimentos cada vez mais complexas, o sistema HACCP em si, e apesar de ser o único com enquadramento legal, começa a revelar-se débil na forma como garante a segurança das cadeias alimentares, uma vez que se suporta numa análise de perigos não intencionais/involuntários e descora a intencionalidade de uma contaminação e/ou adulteração. Os casos mediáticos de contaminação e/ou adulteração vieram reforçar a importância da adoção de medidas de mitigação e eliminação de ameaças e vulnerabilidades relativas a atos intencionais de contaminação ou fraude.

A implementação de um sistema de defesa alimentar proporciona às organizações uma maior resiliência à contaminação e/ou adulteração intencional, fraude alimentar e outras formas de ataque bem como uma maior proteção da reputação e imagem das organizações.

Sistemas de defesa alimentar e de prevenção da fraude alimentar acrescentam robustez e coesão à cadeia de fornecimento de alimentos e como tal, devem ser parte integrante da cadeia de fornecimento de alimentos. É assim essencial aumentar a consciencialização dos profissionais e operadores do sector alimentar para o tema.

\section{AGRADECIMENTOS}

Este trabalho é financiado por Fundos Nacionais através da FCT Fundação para a Ciência e a Tecnologia no âmbito do projeto UID/ Multi/50016/2019.

\section{REFERÊNCIAS BIBLIOGRÁFICAS}

1. World Health Organization. The World Health Report 2007: A Safer Future. Glob Public Health [Internet]. 2007;96. Available from: http://www.who.int/whr/2007/ whr07_en.pdf?ua=1.

2. USDA. Food Safety vs . Food Defense : Differences and Similarities. United States Department of Agriculture. Cairo; 2008. p. 1-26.

3. Detlefsen C, King CH (Hal). Welcome to the Interactive Food Defense Workshop with Government \& Industry Representatives at the 2012 Food Safety Summit and Introduction of Panelists. Food Drug Adm [Internet]. 2012;1-5. Available from: https:// www.fda.gov/Food/NewsEvents/WorkshopsMeetingsConferences/ucm296729.htm. 4. Dalziel GR. Food defence incidents 1950-2008: A Chronology And Analysis Of Incidents Involving The Malicious Contamination Of The Food Supply Chain.Technical Report. [Internet]. Singapore; 2009. Available from: http://www.food-defense.it/1/ upload/rsis_food_defence_170209.pdf.

5. USDA. Food Defense Plan Survey [Internet]. United States Department of Agriculture. 2016 [cited 2017 Sep 6]. Available from: https://www.fsis.usda.gov/wps/portal/fsis/ topics/food-defense-defense-and-emergency-response/functional-food-defense-plan/
functional-plans/!ut/p/a1/04_Sj9CPykssy0xPLMnMzOvMAfGjzOINAg3MDC2dDbwMDIHQ08842MTDy88YKAxUElmswN_SOA2olCzQ39vZ2cDCz5hl_TiAowEh_V5EWGB. 6. BSI. PAS 96:2017 Guide to protecting and defending food and drink from deliberate attack. Br Stand Inst [Internet]. 2017; Available from: https://www.food.gov.uk/sites/ default/files/media/document/pas962017_0.pdf.

7. Bogadi NP, Banović M, Babić I. Food defence system in food industry: perspective of the EU countries. J fur Verbraucherschutz und Leb. 2016;11(3):217-26.

8. European Parliament. REGULATION (EC) No 178/2002 OF THE EUROPEAN PARLIAMENT AND OF THE COUNCIL of 28 January2002 laying down the general principles and requirements of food law, establishing the European Food SafetyAuthorityand laying down procedures in matters of food safety. Off $\mathrm{J}$ Eur Communities [Internet]. 2002;31:1-24. Available from: http://eur-lex.europa.eu/legalcontent/PT/TXT/PDF/?uri=CELEX:32002R0178\&from=EN.

9. Assembleia da Republica. DL n.o 28/84, de 20 de Janeiro [Internet]. Serie I. n.0188, editor. Diário da Republica. 1984 [cited 2017 Aug 26]. Available from: http://www.pgdlisboa.pt/leis/lei_print_articulado.php?tabela=leis\&artigo_ $i d=\& n i d=172 \&$ nversao $=\&$ tabela $=$ leis.

10. IUFoST Scientific Information Bulletin. Short summary on food defense. Int Union Food Sci Technol. 2007;(September):1-7.

11. Spink J, Moyer DC. Defining the Public Health Threat of Food Fraud [Internet]. Vol. 76, Journal of Food Science. 2011 [cited 2017 Jul 2]. p. R157-63. Available from: http://doi.wiley.com/10.1111/j.1750-3841.2011.02417.x.

12. IFS Management GmbH. IFS Food v . 6 Food Defense Guidelines for Implementation. IFS Management GmbH. Berlin; 2012. p. 16.

13. European Commission. Commission launches Knowledge Centre to boost food quality and step up the fight against food fraud [Internet]. European Commission Press release database. 2018 [cited 2018 Apr 4]. Available from: http://europa.eu/ rapid/press-release_IP-18-1744_en.htm.

14. Food Standard Agency. What is food crime and how does it differ from food fraud? [Internet]. Food Standard Agency. 2016 [cited 2017 Jul 2]. Available from: https://www.food.gov.uk/enforcement/the-national-food-crime-unit/what-is-foodcrime-and-food-fraud.

15. Manning L, Soon JM. Food Safety, Food Fraud, and Food Defense : A Fast Evolving Literature. J Food Sci. 2016;81(4):823-34.

16. PwC. Food Fraud Vulnerability Assessment and Mitigation. PricewaterhouseCoopers. 2016;1-37.

17. Pereira FS. Food Defense e Fraude Alimentar: a realidade nacional e internacional. SGS Semin Terror e Fraud Aliment. 2017;71-99.

18. Severino P, Almeida D. Food Defense- Sistemas de gestão contra o terrorismo alimentar. Engebook- Conteudos Eng e Gestão. Publindust. 2017;1-142.

19. World Health Organization. Terrorist Threats to Food Guidance for Establishing and Strengthening Prevention and response systems. Food Saf Dep World Heal Organ [Internet]. 2002;46. Available from: http://apps.who.int/iris/ bitstream/10665/42619/1/9241545844.pdf.

20. FDA. Food Safety and Security : Operational Risk Management Systems Approach. Dep Heal Hum Serv US Food Drug Adm Cent Food Saf Appl Nutr. 2001;(November). 21. USDA. Elements of a Functional Food Defense Plan [Internet]. United States Department of Agriculture. 2016 [cited 2017 Jul 25]. Available from: https://askfsis.custhelp.com/app/ answers/detail/a_id/1032/ /elements-of-a-functional-food-defense-plan.

22. Lindstrom E. Understanding and Preparing for FSMA's HARPC Requirements [Internet]. Food Processing. 2016 [cited 2017 Aug 21]. Available from: http://www. foodprocessing.com/articles/2016/fdsr-harpc-fsma-requirements/?start=1.

23. King, Hal and Ades G. Hazard Analysis and Risk-Based Preventive Controls (HARPC): The New GMP for Food Manufacturing [Internet]. Food Safety Magazine. 2015 [cited 2017 Aug 19]. Available from: http://www.foodsafetymagazine.com/magazinearchive1/octobernovember-2015/hazard-analysis-and-risk-based-preventive-controlsharpc-the-new-gmp-for-food-manufacturing/.

24. FDA. CARVER + SHOCK PRIMER An overview of the CARVER PLUS SHOCK method for food sector vulnerability assessments. Food Drug Adm [Internet]. 2009;1-14. Available from: https://www.fda.gov/food/fooddefense/fooddefenseprograms/ucm376791.htm. 
25. Spink J. GFSI Direction on Food Fraud and Vulnerability Assessment (VACCP) [Internet]. Food Fraud Initiative, Michigan State University. 2014 [cited 2017 Aug 13]. Available from: http://foodfraud.msu.edu/2014/05/08/gfsi-direction-on-food-fraudand-vulnerability-assessment-vaccp/.

26. GFSI. GFSI Position On Mitigating The Public Health Risk Of Food Fraud. Global Food Safety Initiative [Internet]. 2014;1-9. Available from: http://www.mygfsi.com/files/ Technical_Documents/Food_Fraud_Position_Paper.pdf.

27. SSAFE. Food Fraud Vulnerability Assessment Tool [Internet]. SSAFE. 2019 [cited 2019 Nov 17]. Available from: http://www.ssafe-food.org/our-projects/?proj=365.

28. ASAE. Comunicado no 1 - Carne de cavalo [Internet]. Autoridade de Segurança Alimentar e Económica. 2013 [cited 2017 Jul 24]. Available from: http://www.asae.pt/ pagina. $a$ sp $x$ ? $=3 \&$ back $=1$ \&id $=12728$.

29. Spink J, Embarek P Ben, Savelli CJ, Bradshaw A. Global perspectives on food fraud: results from a WHO survey of members of the International Food Safety Authorities Network (INFOSAN). npj Sci Food [Internet]. 2019;3(1):1-5. Available from: http://dx.doi.org/10.1038/s41538-019-0044-x.

30. Spink J, Elliott C, Dean M, Speier-Pero C. Food fraud data collection needs survey. npj Sci Food [Internet]. 2019;3(1):1-8. Available from: http://dx.doi.org/10.1038/ s41538-019-0036-x.

31. Davidson RK, Antunes W, Madslien EH, Belenguer J, Gerevini M, Torroba Perez T, et al. From food defence to food supply chain integrity. Br Food J. 2017;119(1):52-66. 